\title{
Aprendiendo análisis estratégico y trabajando la responsabilidad medioambiental con la Unidad de Medio Ambiente de la Universitat Politècnica de València
}

\author{
de-Miguel Molina, María ${ }^{a}$; Catalá-Pérez, Daniel ${ }^{b}$; de-Miguel-Molina, Blanca ${ }^{\mathfrak{c}}$ y \\ Peiró-Signes, Ángel d \\ aUniversitat Politècnica de València, mademi@omp.upv.es, bUniversitat Politècnica de València, dacape@upv.es, \\ 'Universitat Politècnica de València, bdemigu@omp.upv.es y dUniversitat Politècnica de València, \\ anpeisig@omp.upv.es
}

\begin{abstract}
The aim of this activity was to show the real case of a public service by means of its manager in order to explain how important is the strategic analysis phase for the complete strategy of an organization. In this case, an Environmental Management System is a perfect example, due to its transversality, to understand how stakeholders are involved in the organization's strategy, how internal and external factors can influence it, and the necessary flexibility that the organization's strategy itself requires to be frequently adapted to the fast changes that society demands. Moreover, we can give an example of the sustainable development goals and to enhance the general competence "environmental responsibility".
\end{abstract}

Keywords: strategic analysis, public service, environmental management system, adaptative strategy, sustainable development goals, environmental responsibility.

\section{Resumen}

El objetivo de esta actividad fue mostrar el caso real de un servicio público a través de su directora para explicar la importancia del análisis estratégico en la estrategia integral de una organización. En este caso, el Sistema de Gestión Medioambiental es un ejemplo perfecto, dada su transversalidad, para comprender la influencia que los grupos de interés tienen en la estrategia de la organización, cómo la influyen los factores internos y externos, y la necesaria flexibilidad que la misma estrategia de la organización precisa para adaptarse frecuentemente a los rápidos cambios que demanda la sociedad. Además, esta práctica nos permitió dar un ejemplo práctico sobre la búsqueda de los objetivos de desarrollo sostenible e incidir en la competencia transversal "responsabilidad medioambiental".

Palabras clave: análisis estratégico, servicio público, sistema de gestión medioambiental, estrategia adaptativa, objetivos de desarrollo sostenible, responsabilidad medioambiental. 


\section{Introducción}

El análisis estratégico es la primera fase de cualquier proceso de dirección estratégica (Figura 1). En esta etapa, se establecerán o re-establecerán los objetivos y misión de la organización, en función de la información primaria y secundaria que extraigamos (de Miguel et al, 2017).

Pero, además, el entorno actual obliga a adaptarse continuamente a distintos cambios. Mulgan (2009) habla de la necesidad de una "estrategia adaptativa", en la que los distintos elementos de la dirección estratégica se van adaptando al entorno y, para ello, es esencial que la organización analice frecuentemente no sólo sus elementos internos sino también ese entorno en el que opera. Y el medio ambiente, en especial, es un buen ejemplo de ello.

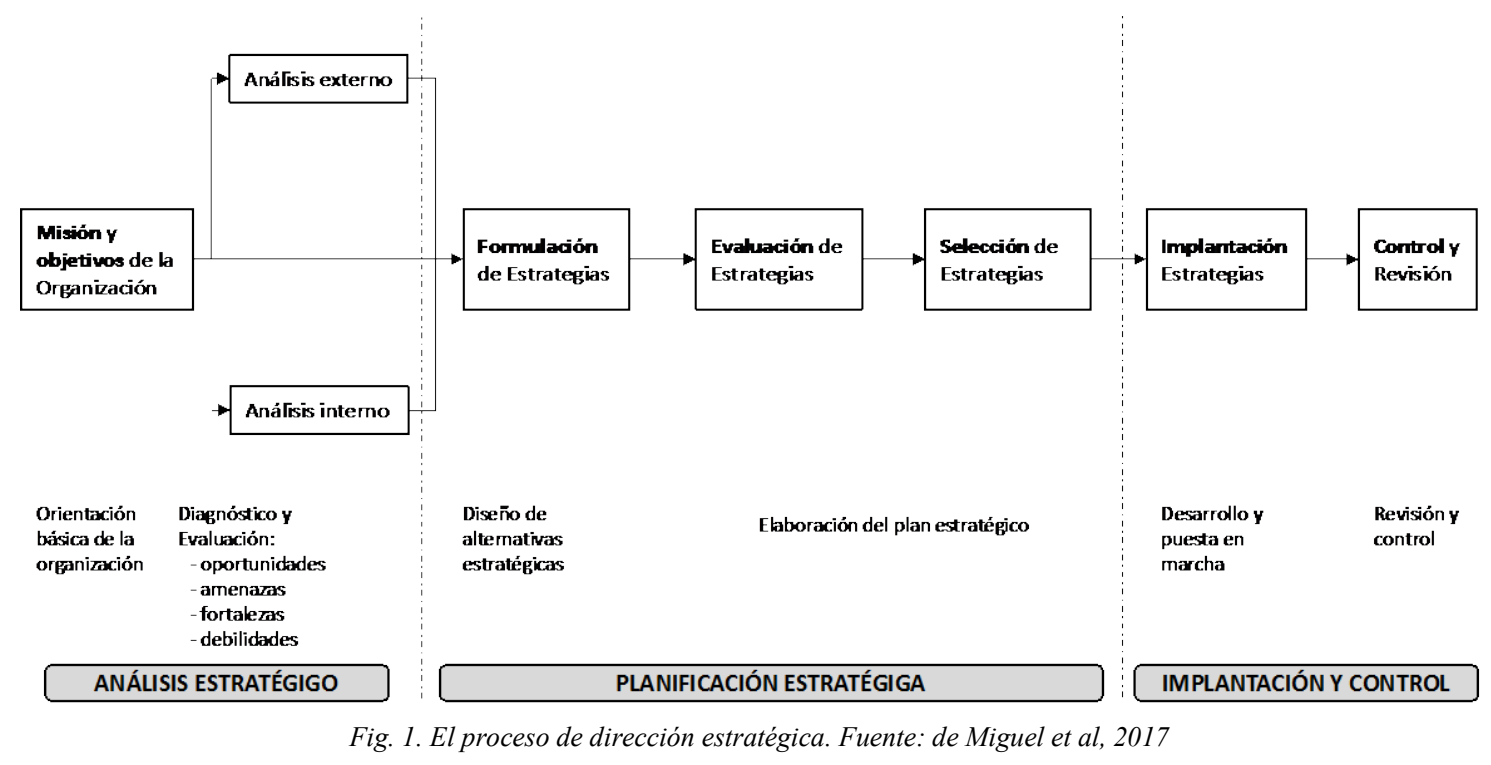

En el curso 2018-2019, tuvimos la oportunidad de realizar una práctica en el aula con $\mathrm{D}^{\mathrm{a}}$ Cristina Martí Barranco, Jefa de la Unidad de Medio Ambiente (UMA) de la UPV, en la asignatura Dirección Estratégica de Organizaciones Públicas (De Miguel et al, 2017), obligatoria de $3^{\circ}$ curso, del Grado en Gestión y Administración Pública de la Facultad de Administración y Dirección de Empresas de la Universitat Politècnica de València (UPV).

Cuando planificamos esta práctica, pensamos que la mejor opción sería encuadrarla en el tema "Dirección estratégica", puesto que esta Unidad realiza una actividad transversal dentro de la UPV y el tipo de presentación que se iba a realizar permitía que los alumnos pudieran utilizar una de las herramientas fundamentales en cualquier análisis estratégico. El análisis DAFO (Debilidades, Amenazas, Fortalezas y Oportunidades) es, en este sentido, una herramienta sencilla en su aplicación pero muy potente en cuanto a los resultados que permite obtener sobre la situación interna y externa de una organización antes de proceder a la fase de planificación estratégica. 
Al mismo tiempo, se plantearon otros objetivos de formación transversal como potenciar la responsabilidad medioambiental, a través de la identificación de objetivos de desarrollo sostenible, analizando un ejemplo real con su propio gestor.

\section{Objetivos}

Entre la Jefa de la UMA y los profesores planteamos como objetivos:

- Que los alumnos fuesen capaces de distinguir entre la propia organización (UPV, UMA) y el entorno.

- Reunir la información necesaria, a partir de la exposición de la Jefa de la UMA, así como de la información disponible en la página web de la UMA.

- Elegir y clasificar la información relevante para incluir en el análisis clásico de Debilidades, Amenazas, Fortalezas y Oportunidades (DAFO).

- Identificar los aspectos positivos y negativos de dicha información (F-O frente a D-A).

- Relacionar la información relevante con los objetivos de desarrollo sostenible (ODS) de Naciones Unidas (Naciones Unidas, 2016).

- Además, surgió la oportunidad de incidir en estos objetivos ya que la semana anterior la propia UMA había establecido la "Semana de los ODS", en el marco del 50 Aniversario de la UPV, por lo que los alumnos tenían un conocimiento previo de ellos.

- Al mismo tiempo, y sin que estuviese previsto en la evaluación del alumno, esta práctica permitió incidir en la competencia transversal "responsabilidad medioambiental", como la conciencia del impacto que tiene nuestra actividad o falta de ella en otras especies, la naturaleza o las futuras generaciones (UPV, 2012).

\section{Desarrollo de la innovación}

La Jefa de la Unidad hizo una exposición muy participativa de manera que la información que pudiesen extraer los alumnos fuera lo más completa posible. En primer lugar, se dejó clara la misión de la Unidad de Medio Ambiente (UMA) dentro de la UPV:

"Desarrollar tareas de información y sensibilización ambiental.

Gestionar los aspectos ambientales directos e indirectos generados por las actividades universitarias.

Implantar, certificar y mantener el sistema de gestión ambiental según la norma ISO 14001 y el reglamento EMAS.

Y todo ello con objeto de controlar y minimizar el impacto que la universidad tiene sobre el medio ambiente" (Unidad de Medio Ambiente de la UPV, 2019). 
Seguidamente, se explicaron todas las actividades que realiza la UMA, los grupos de interés con los que trabaja, los servicios que ofrece, etc. Asimismo, se recabó información de los alumnos a través de una encuesta para que aportasen ideas con las que mejorar el servicio de la UMA.

Una vez realizada la exposición, los alumnos tenían que realizar un análisis DAFO por equipos, que los profesores recogieron al final de la sesión. Con los distintos análisis, los profesores integraron toda la información y se la enviaron a la Jefa de la UMA para que la revisara. En la siguiente sesión, y ya solo con los profesores, se mostraron las conclusiones para compararla con la información analizada por ellos. Además, se identificaron los ODS presentes en la actividad de la UMA.

De este modo, a través de un caso real se pudieron no solo trabajar competencias transversales como la responsabilidad medioambiental, sino también incidir en los objetivos de aprendizaje de este tema:

- Conocer los conceptos básicos de la Dirección estratégica.

- Conocer el proceso de la planificación estratégica y las particularidades propias de las administraciones públicas.

- Analizar estrategias formuladas en distintas organizaciones públicas.

\section{Resultados}

El resultado final del análisis integrado se muestra en la Figura 2. Como se puede observar, la información se clasificó de acuerdo con el clásico análisis DAFO y se mostró de la manera más sencilla posible.

Uno de los aspectos destacados de la experiencia fue que la revisón del DAFO integrado (Figura 2) suscitó un interesante debate acerca de la consideración de ciertos elementos como negativos o positivos, tanto en el ámbito interno como externo, en función de los objetivos o de la perspectiva adoptada para su análisis respectivamente.

Ello permitió que, tras la realización de la práctica, los alumnos identificaran más claramente la mayoría de los conceptos clave de la dirección estratégica y reconocieran la importancia de la correcta definición de elementos como la misión, visión, valores, objetivos, etc... para ser capaces de realizar un análisis posterior lo más valioso y certero posible. 

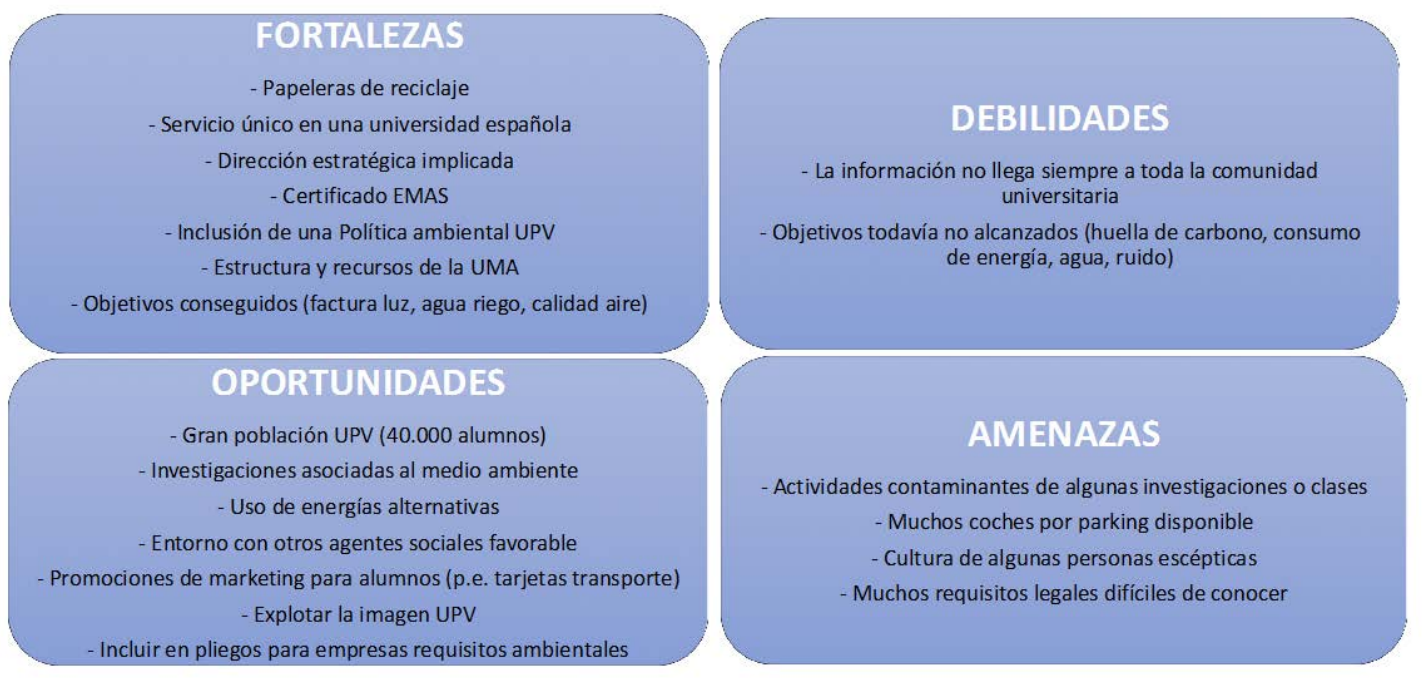

Fig. 2. Análisis DAFO de la Unidad de Medio Ambiente de la UPV. Fuente: elaboración propia.

Además, se pidió a los alumnos que identificasen qué objetivos de desarrollo sostenible encontraban en la actividad realizada por la UMA de la UPV. Este análisis permitió, al mismo tiempo, incidir en la competencia transversal "responsabilidad medioambiental".

En este caso, se identificaron principalmente dos (De Miguel y Catalá, 2019):

- Consumo sostenible (objetivo 12): puesto que su misión se basa en concienciar y ayudar a la comunidad universitaria a que piense de una manera sostenible a la hora de utilizar la energía y los recursos. Además, pone a disposición de toda la universidad herramientas que faciliten esta labor, como los diversos contenedores de reciclaje.

- Prevenir los efectos del cambio climático (objetivo 13): ya que, con su aportación, la comunidad universitaria que integra un volumen importante de personas puede contribuir localmente a reducir el cambio climático, lo que seguro también contribuye al efecto global.

Después de realizar y analizar esta práctica, se les explicó a los alumnos el tema correspondiente (de Miguel et al, 2017) haciendo uso para ello de un mapa mental en el que constantemente se utilizó el caso que se había visto para explicar los distintos apartados lo que nos permitió afianzar los conceptos (Figura 3): 


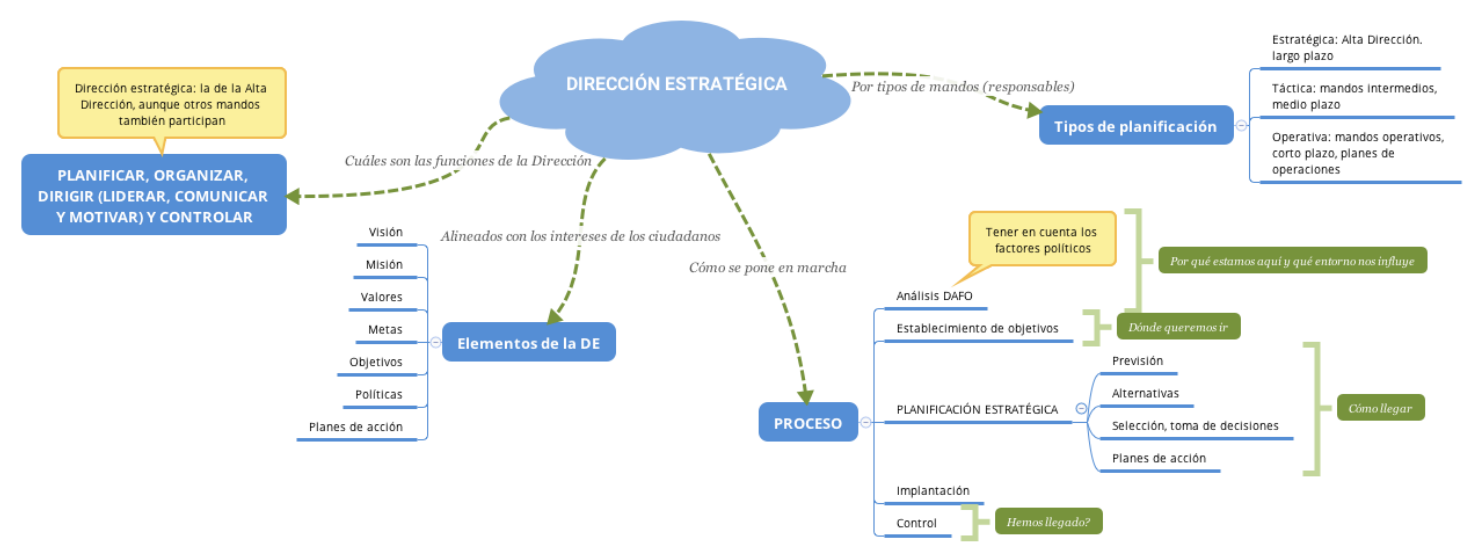

Fig. 3. La Dirección estratégica. Fuente: de Miguel et al, 2017.

\section{Conclusiones}

La oportunidad que se nos brindó por la UMA de la UPV fue una experiencia inmejorable para que los alumnos viesen los conceptos de Dirección estratégica aplicados en un caso real. Además, es un tema que les es cercano y del que pudieron opinar en todo momento. De hecho, uno de los aspectos destacados de la experiencia fue el debate que se abrió durante y después de la exposición de la Jefa de Unidad de la UMA. Los alumnos pudieron trasladar algunas de las situaciones y conceptos planteados a sus entornos más próximos, comparando, por ejemplo, la actuación de la UMA con la de sus ayuntamientos.

De este modos conseguimos que conociesen los conceptos básicos de la Dirección estratégica de una manera práctica, pudiesen conocer el proceso de la planificación estratégica seguido por un gestor público, reflexionasen sobre las particularidades propias de una administración pública y pudiesen analizar las estrategias medioambientales formuladas por la UPV.

Al mismo tiempo, ayudamos al mejor conocimiento del trabajo que realiza la UPV por la sostenibilidad y el cuidado del medio ambiente. Y se pudo ver un ejemplo práctico de la aplicación de los objetivos de desarrollo sostenible.

\section{Agradecimientos}

Agradecemos a D ${ }^{a}$ Cristina Martí Barranco, Jefa de la Unidad de Medio Ambiente (UMA) de la UPV, su disposición e implicación para poder realizar esta práctica en el aula.

\section{Referencias}

DE-MIGUEL-MOLINA, M.; BAÑÓN GOMIS, A.J.; CATALÁ-PÉREZ, D. (2017). Management para las Administraciones públicas. Valencia: Editorial Universitat Politècnica de València. 
DE-MIGUEL-MOLINA, M.; CATALÁ PÉREZ, D. (2019). Análisis estratégico de la Unidad de Medio Ambiente de la UPV. Objeto de Aprendizaje, UPV.

MUlGAN, G. (2009). The Art of Public Strategy. Mobilizing Power and Knowledge for the Common Good. Oxford University Press.

NACIONES UNIDAS. Objetivos de desarrollo sostenible.

https://www.un.org/sustainabledevelopment/es/objetivos-de-desarrollo-sostenible/

[Consulta: 8 de mayo de 2019]

UNIDAD DE MEDIO AMBIENTE DE LA UNIVERSITAT POLITECNICA DE VALENCIA. Política ambiental. http://www.upv.es/entidades/AMAPUOC/indexes.html [Consulta: 12 de mayo de 2019]

UPV. Competencias transversalesUPV. http://www.upv.es/contenidos/COMPTRAN/ [Consulta: 14 de mayo de 2019] 